Conclusions: Results of this post hoc analysis suggest that, in men with RA, there was no impact of BMI on ETN efficacy. In women, there was evidence of a transient negative impact of $\mathrm{BMI} \geq 30 \mathrm{~kg} / \mathrm{m}^{2}$ on ETN efficacy in open-label periods, but it diminished by the end of the induction period and was not consistent across trials. There was no evidence of BMI effect in men or women during the double-blind periods.

Acknowledgements: Sponsored by Pfizer Inc.

Disclosure of Interest: R. Alten Grant/research support from: Pfizer, Speakers bureau: Pfizer, E. Mysler Consultant for: Abbvie, BMS, Pfizer, Roche, Pharma, GEMMA, A. Wajdula: None declared, H. Jones Shareholder of: Pfizer, Employee of: Pfizer, R. Pedersen Shareholder of: Pfizer, Employee of: Pfizer, L. Marshall Shareholder of: Pfizer, Employee of: Pfizer DOI: 10.1136/annrheumdis-2018-eular.3253

\section{FRI0035 DISEASE REMISSION IS MORE COMMON BUT LESS STRINGENT IN ANTI-CITRULLINATED PROTEIN ANTIBODY-POSITIVE PATIENTS WITH EARLY RHEUMATOID ARTHRITIS TREATED WITH CONVENTIONAL SYNTHETIC DISEASE MODIFYING DRUGS}

A. Manzo, S. Bugatti, G. Zanframundo, F. Benaglio, G. Sakellariou, C. Montecucco, R. Caporali. Early Arthritis Clinic, Division of Rheumatology, University of Pavia, IRCCS Policlinico San Matteo Foundation, Pavia, Italy

Background: Early diagnosis and goal-steered treatment strategies allow the achievement of disease remission in a significant proportion of patients with early rheumatoid arthritis (RA). Autoantibodies such as anti-citrullinated protein autoantibodies (ACPA) identify a subset of patients with a common pathogenetic background and more severe course of the disease. However, whether autoantibodies also impact the response to conventional synthetic disease modifying anti-rheumatic drugs (csDMARDs) remains object of debate.

Objectives: To investigate the frequency and the characteristics of disease remission in relation to the autoantibody status in patients with early RA treated with csDMARDs.

Methods: The study population consisted of 578 early RA patients $(<12$ months of symptoms) consecutively recruited at our Early Arthritis Clinic, treatment-naïve at inclusion and prospectively followed-up at regular intervals upon initiation of therapy with methotrexate (MTX) aimed at the achievement of low disease activity (28-joints disease activity score [DAS28] $\leq 3.2$ ). ACPA and rheumatoid factor (RF) were centrally determined on baseline sera. The frequency of remission according to the DAS28 $(<2.6)$ and to the simplified disease activity index (SDAI $\leq 3.3)$ over the first 12 months stratified by the autoantibody status was assessed by Cox regression. The following characteristics were further analysed: time to remission, swollen and tender joint count, and acute phase reactants at remission.

Results: 533/578 (92.2\%) patients completed a follow-up of at least 12 months. Patients fulfilled the 2010 classification criteria for RA ( $81 \%$ also fulfilled the 1987 criteria) and had short disease duration at inclusion (median [IQR] 15.6 [9.4-27.8] weeks). Collectively, $53.9 \%$ and $44 \%$ of the patients achieved DAS28 and SDAI remission, respectively, at least once over the first 12 months of treatment. After adjusting for age, gender, symptoms' duration, baseline disease activity, MTX starting dose and prednisone co-medication, ACPA positivity was associated with slightly increased hazards of achieving both DAS28 (HR [95\% Cl] 1.24 [1.011.63]) and SDAI remission (HR [95\% Cl] 1.36 [1.01-1.85]) (figure 1A, B). However, irrespective of the remission criterion, ACPA-positive patients had higher numbers of residual swollen joints while being in remission, particularly in association with high levels of RF (>3 ULN) (figure 1C, D). Furthermore, remission was delayed in RF-high ACPA-positive patients compared to RF-low (figure 1E, F). Other features such as joint tenderness and acute phase reactants did not show significant differences among different serological subgroups.
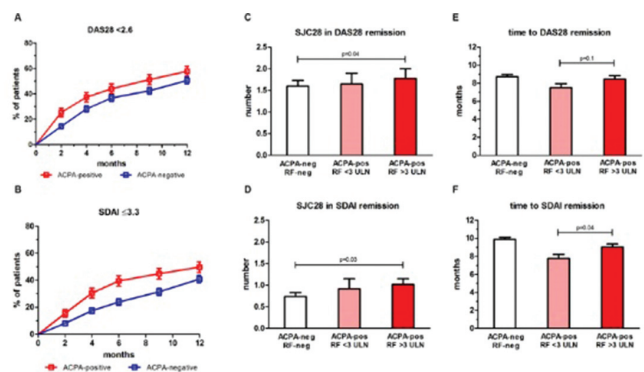

Disclosure of Interest: None declared

DOI: 10.1136/annrheumdis-2018-eular.6881
Conclusions: Early diagnosis and initial treatment with MTX result in high remission percentages in RA patients regardless of autoantibody positivity. However remission appears less stringent in ACPA-positive patients, particularly when RF is also high. These findings indicate that current treatment approaches may be insufficient at effectively suppressing joint inflammation in autoantibody-positive patients.

Disclosure of Interest: None declared

DOI: 10.1136/annrheumdis-2018-eular.7565

\section{FRI0036 $\quad$ SUSTAINED REMISSION RELATED FACTORS IN PATIENTS WITH RHEUMATOID ARTHRITIS: IS IT POSSIBLE TO PREDICT SUSTAINED REMISSION?}

S. Yilmaz-Oner, U. Gazel, M. Can, P. Atagunduz, H. Direskeneli, N. Inanc. Rheumatology, Marmara University, School of Medicine, Istanbul, Turkey

Background: The management of RA have been changed during past decades and sustained remission (SR) is the ultimate goal to halt joint damage and prevent the accrual of disability. Factors predicting SR are essential to individualise treatment and recognise patients that have an opportunity to taper medications in daily practice.

Objectives: To determine baseline predictors of sustained remission and comparison of its predictability by different remission criteria.

Methods: A total of 428 consecutive patients with RA visiting our outpatient clinic routinely between September 2012-2013 were evaluated. Seventy seven of these patients satisfying the DAS28 remission $(<2.6)$ within the time frame were enrolled and followed-up prospectively for $62.2 \pm 9.9$ months. Of these fulfiling the DAS28 remission $\geq 6$ months (sustained remission) and shorter (non-sustained remission:N-SR) were compared in terms of baseline demographic and clinica data and the presence of anxiety, depression, fibromyalgia and fatigue to deter mine possible predictors of SR. At enrollment, first and fifth years, the DAS28 SDAI and Boolean remission rates of patients were determined and compared with regard to DAS28 remission visit counts throughout the follow-up. We also assessed the difference between the SDAI and Boolean remission rates at initial, first and fifth years' visits.

Results: Of these 77 patients, 63 were in SR and 14 were in N-SR. Lower baseline DAS28 and HAQ scores $(p=0.045 ; p=0.026$, respectively) and anti-CCP positivity $(p=0.035)$ were positive predictors of $S R$. Although the presence of anxiety depression, fibromyalgia and fatigue were lower in SR group, there was no statistical significance.

DAS28 remission visit counts of patients in Boolean $(n=32)$ and DAS28 $(n=77)$ remission at enrollment $(5.7 \pm 3.2$ vs $5.4 \pm 3.1 ; p=0.995)$ were not different. Similarly, no difference was found between patients in SDAI $(n=38)$ and DAS28 remis sion $(5.6 \pm 3.3$ vs $5.4 \pm 3.1 ; p=0.769)$. Patients meeting the DAS28 criteria $(n=77$ $100 \%)$ reduced $64 \%(n=50)$ at first and $42.6 \%(n=29)$ at fifth years. Patients satisfying SDAl and Boolean criteria were $49 \%$, $44 \%$ vs $32.4 \%(n=22)$ and $41 \%, 28 \%$ vs $20.6 \%(n=14)$, respectively.

If the duration of SR is considered as 6 months, the remission rates of SDAI were not different between patients at inclusion and fifth years but Boolean remission rates differed significantly and if it is accepted as $\geq 12$ months, the SDAI and Boolean remission rates were not different than at fifth year visit.

Conclusions: Low DAS28 and HAQ score at baseline and anti-CCP positivity were positive predictors of SR. Although the presence of anxiety, depression, fibromyalgia and fatigue were lower in SR group, there was no statistical significance. Compared to the DAS28, remission determined by the Boolean and especially SDAI criteria continued consistently in long term.

\section{REFERENCES}

[1] Castrejón I, Dougados M, Combe B, et al. Prediction of remission in a French Early Arthritis Cohort by RAPID3 and other core data set measures, but not by the absence of rheumatoid factor, anticitrullinated protein antibodies, or radiographic erosions. J Rheumatol. 2016 Jul;43(7):128591.

[2] Radner H, Alasti F, Smolen JS, et al. Physical function continues to improve when clinical remission is sustained in rheumatoid arthritis patients. Arthritis Res Ther. 2015 Aug 11;17:203.

Acknowledgements: None. 